\title{
BOUNDEDNESS AND DIMENSION FOR WEIGHTED AVERAGE FUNCTIONS ${ }^{1}$
}

\author{
DAVID P. STANFORD
}

\begin{abstract}
The paper considers a weighted average property of the type $u\left(x_{o}\right)=\left(\int_{B} u w d x\right) /\left(\int_{B} w d x\right), B$ a ball in $E^{n}$ with center $x_{o}$. A lemma constructing such functions is presented from which it follows that if $n=1$ and the weight function $w$ is continuously differentiable but is not an eigenfunction of the 1-dimensional Laplace operator, then $u$ is constant. It is also shown that if $w$ is integrable on $E^{n}$ and $u$ is bounded above or below, $u$ is constant.
\end{abstract}

Let $D$ be a region in $n$-dimensional Euclidean space $E^{n}$. Following A. K. Bose [1] we say a weight function on $D$ is a nonnegative, locally integrable function on $D$ whose integral over any closed ball lying in $D$ is positive.

A real valued function $u$ has the weighted average property with respect to the weight function $w$ on $D$ if $u w$ is locally integrable on $D$ and, for every closed ball $B$ lying in $D$ with center at $x_{0}, u\left(x_{0}\right)$ $=\left(\int_{B} u w d x\right) /\left(\int_{B} w d x\right)$. We denote by $S(w, D)$ the collection of functions satisfying the weighted average property with respect to $w$ in $D$. $S(w, D)$ is a real vector space containing the constants.

Bose has shown in [1], [2] and [3]:

(i) If $n>1$ and $w$ is an eigenfunction of the Laplace operator $\Delta$, then the dimension, $\operatorname{dim} S(w, D)$, of $S(w, D)$ is $\infty$.

(ii) If $n=2, w$ is in $C^{1}(D)$, and $w$ is not an eigenfunction of $\Delta$, then $1 \leqq \operatorname{dim} S(w, D) \leqq 2$.

(iii) For $n>2$, there is a weight function $w$ on $E^{n}$ which is not an eigenfunction of $\Delta$ but for which $\operatorname{dim} S\left(w, E^{n}\right)=\infty$.

(iv) If $w$ is a bounded continuous weight function on $E^{n}$ with a positive lower bound and $u$ is a bounded function satisfying the weighted average property with respect to $w$, then $u$ is constant.

I prove in this note:

THEOREM 1. If $D$ is an interval in $E^{1}$ and if $w$ is a weight function belonging to $C^{1}(D)$ which is not an eigenfunction of the 1-dimensional

Received by the editors March 10, 1969.

AMS Subject Classification. Primary 3111.

Key Words and Phrases. Eigenfunction of Laplace operator, dimension of space of functions satisfying a weighted average property, weight function integrable over $E^{n}$, bounded function satisfying a weighted average property.

1 This research was supported in part by the U. S. Army Research Office in Durham, N. C. 
Laplace operator, then $S(w, D)$ contains only the constants.

THEOREM 2. If $w$ is a weight function integrable over $E^{n}, u$ is in $S\left(w, E^{n}\right)$, and $u$ is bounded either above or below, then $u$ is constant.

The proof of Theorem 1 is based on the following;

Lemma. Let $w_{1}(x)$ be a weight function on $D_{1} \subset E^{\alpha}, w_{2}(y)$ a weight function on $D_{2} \subset E^{\beta}, u_{1}(x) \in S\left(w_{1}, D_{1}\right)$, and $u_{2}(y) \in S\left(w_{2}, D_{2}\right)$. Then $u(x, y)=u_{1}(x) u_{2}(y)$ belongs to $S\left(w, D_{1} \times D_{2}\right)$, where $w(x, y)=w_{1}(x) w_{2}(y)$.

Proof. It is clear that $w$ is a weight function and $u w$ is locally integrable on $D_{1} \times D_{2}$. For $x$ in $E^{n}, 0<r<s$, we denote by $B(x, r)$ the closed ball centered at $x$ of radius $r$, and by $A(x, r, s)$ the closed annulus centered at $x$ of radii $r$ and $s$. Suppose $\left(x_{0}, y_{0}\right) \in D_{1} \times D_{2}$, and $r>0$ such that $B\left(\left(x_{0}, y_{0}\right), r\right) \subset D_{1} \times D_{2}$. For positive integers $m$ and $p$ with $1 \leqq p \leqq 2^{m}-1$, let

$$
\begin{gathered}
r_{m, p}=r\left(1-p^{2} / 2^{2 m}\right)^{1 / 2}, \\
C(m, p)=B\left(x_{0}, r_{m, p}\right) \times A\left(y_{0},(p-1) r / 2^{m}, p r / 2 m\right),
\end{gathered}
$$

and

$$
S_{m}=\cup_{p} C(m, p) \text {. }
$$

The following three statements follow from tedious but straightforward calculations:

(a) The $(\alpha+\beta)$-Lebesgue measure of $C(m, p) \cap C(m, q)$ is zero for $p \neq q$.

(b) $S(m) \subset S(m+1), m \geqq 1$.

(c) Interior $B\left(\left(x_{0}, y_{0}\right), r\right) \subset U_{m} S(m) \subset B\left(\left(x_{0}, y_{0}\right), r\right)$.

It is easily seen that

$$
u_{2}\left(y_{0}\right) \int_{A\left(y_{0}, s, t\right)} w_{2}=\int_{A\left(y_{0}, \varepsilon, t\right)} u_{2} w_{2} \text { whenever } B\left(y_{0}, t\right) \subset R_{2} .
$$

Thus, from Fubini's Theorem,

$$
u\left(x_{0}, y_{0}\right) \int_{C(m, p)} w=\int_{C(m, p)} u w \quad \text { for all } m, 1 \leqq p \leqq 2^{m}-1 .
$$

From (a) it follows that $u\left(x_{0}, y_{0}\right) \int_{S(m)} w=\int_{S(m)} u w$. Statement (b) allows us to take limits as $m \rightarrow \infty$, and using (c) also, we obtain

$$
u\left(x_{0}, y_{0}\right) \int_{B\left(\left(x_{0}, y_{0}\right), r\right)} w=\int_{B\left(\left(x_{0}, y_{0}\right) r\right)} u w
$$

which completes the proof. 
Proof of Theorem 1. Suppose $u \in S(w, D)$ and $u$ is not constant. Let $w_{2} \equiv 1$ on $E^{1}$. Then $w(x) w_{2}(y)$ is in $C^{1}\left(D \times E^{1}\right)$ and is not an eigenfunction of $\Delta$. Further, $u_{2}(y)=y$ is in $S\left(w_{2}, E^{2}\right)$. Thus, by the lemma, each of $u(x), u_{2}(y)$, and 1 is in $S\left(w(x) w_{2}(y), D \times E^{1}\right)$, and since these functions are linearly independent, Bose's result (ii) is contradicted.

We note that the following statement also follows easily from the lemma to Theorem 1:

If $D \subset E^{n}, n>2$, and $w$ is a weight function on $D$ independent of two of the variables, then $S(w, D)=\infty$.

The author is indebted to the referee for the following proof of Theorem 2, which is shorter than the original.

Proof of Theorem 2. Suppose $u \in S\left(w, E^{n}\right)$ and $u$ is bounded below. Let $K>0$ such that $v=u+K$ is positive. Then $v \in S\left(w, E^{n}\right)$ and, for $y \in E^{n}, R>0, \int_{B(y, R)} v(x) w(x) d x=v(y) \int_{B(y, R)} w(x) d x$. Since $w$ is integrable on $E^{n}$, vw is integrable on $E^{n}$, and, letting $R \rightarrow \infty, v(y)$ $=\int v(x) w(x) d x / \int w(x) d x$, the integrals taken over all of $E^{n}$. Thus $v$ is constant, so $u$ is constant. If $u$ is bounded above, consider $-u$.

\section{REFERENCES}

1. Anil K. Bose, Functions satisfying a weighted average property, Trans. Amer. Math. Soc. 118 (1965), 472-487. MR 31 \#1392.

2. - Functions satisfying a weighted average property. II, Trans. Amer. Math. Soc. 124 (1966), 540-551. MR 34 \#4518.

3. - Generalized eigenfunctions of the Laplace operator and weighted average property, Proc. Amer. Math. Soc. 19 (1968), 55-62. MR $36 \# 4173$.

College of William and Mary 